\title{
PL-1
}

\section{Laparoscopic living donor right hepatectomy regarding the anatomical variation of portal vein: A propensity score matched analysis}

\author{
Jinsoo RHU, Gyu-Seong CHOI*, Jong Man KIM, Jae-Won JOH \\ Department of Surgery, Samsung Medical Center, Seoul, Korea
}

Introduction: This study is designed to analyze the feasibility of laparoscopic living donor right hemihepatectomy in living donors with portal vein variation.

Methods: Living donor liver transplantation cases with right liver graft during the period of January 2014 to September 2019 were included. Computed tomographic angiographies of the donor were three-dimensionally reconstructed, and the anatomical variation of the portal vein was classified. To reduce, selection bias, 1:1 ratio propensity score match analysis between the laparoscopy group and the open group was performed. Surgical and recovery-related outcome as well as portal vein complication-free survival, graft survival and overall survival were analyzed.

Results: After matching, 171 cases in each group from 444 original cases were compared. The laparoscopy group had shorter operation time $(p<0.001)$, smaller number of additional opioids required by the donor $(p<0.001)$, shorter hospital stay $(p<0.001)$. There were no difference in the portal vein complication-free survival $(p=0.16)$, graft survival $(p=0.26)$, and overall survival $(p=0.53)$. While portal vein complication-free survival was inferior in portal vein other than type I $(p=0.01)$, the laparoscopy showed similar portal vein complication-free survival whether the portal vein was type I $(p=0.35)$ or other types $(p=0.30)$.

Conclusions: Laparoscopic living donor right hemihepatectomy can be performed as safely as open surgery regardless of the anatomical variation of portal vein. 